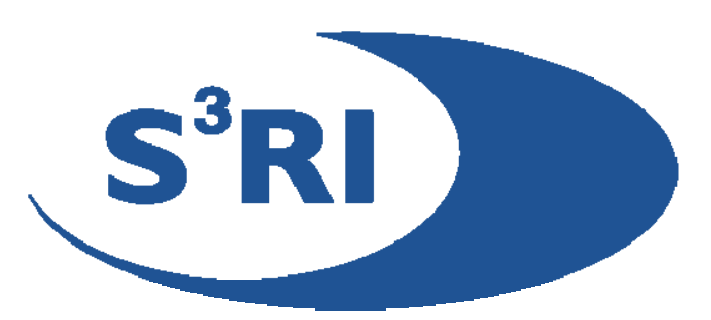

\title{
A Study of Partial $F$ Tests For Multiple Linear Regression MODELS
}

\author{
Wei LiU, MORTAZA JAMSHIDIAN
}

\begin{abstract}
Partial F tests play a central role in model selections in multiple linear regression models. This paper studies the partial $\mathrm{F}$ tests from the view point of simultaneous confidence bands. It first shows that there is a simultaneous confidence band associated naturally with a partial $\mathrm{F}$ test. This confidence band provides more information than the partial $\mathrm{F}$ test and the partial $\mathrm{F}$ test can be regarded as a side product of the confidence band. This view point of confidence bands also leads to insights of the major weakness of the partial $\mathrm{F}$ tests, that is, a partial $\mathrm{F}$ test requires implicitly the linear regression model holds over the entire range of the covariates in concern. Improved tests are proposed and they are induced by simultaneous confidence bands over restricted regions of the covariates. Power comparisons between the partial F tests and the new tests have been carried out to assess when the new tests are more or less powerful than the partial F tests. Computer programmes have been developed for easy implements of these new confidence band based inferential methods. An illustrative example is provided.
\end{abstract}

\section{Southampton Statistical Sciences Research Institute Methodology Working Paper M07/03}




\title{
A STUDY OF PARTIAL $F$ TESTS FOR MULTIPLE LINEAR REGRESSION MODELS
}

\author{
Wei Liu \\ Statistical Sciences Research Institute and School of Maths \\ The University, Southampton, SO17 1BJ, U.K. \\ Mortaza Jamshidian ${ }^{1}$ \\ Department of Maths, California State Univ., Fullerton, CA 92834, U.S.A.
}

September 7, 2005

${ }^{1}$ Mortaza Jamshidian's research has been supported in part by the National Science Foundation Grant DMS-0437258. 


\begin{abstract}
Partial $F$ tests play a central role in model selections in multiple linear regression models. This paper studies the partial $F$ tests from the view point of simultaneous confidence bands. It first shows that there is a simultaneous confidence band associated naturally with a partial $F$ test. This confidence band provides more information than the partial $F$ test and the partial $F$ test can be regarded as a side product of the confidence band. This view point of confidence bands also leads to insights of the major weakness of the partial $F$ tests, that is, a partial $F$ test requires implicitly the linear regression model holds over the entire range of the covariates in concern. Improved tests are proposed and they are induced by simultaneous confidence bands over restricted regions of the covariates. Power comparisons between the partial $F$ tests and the new tests have been carried out to assess when the new tests are more or less powerful than the partial $F$ tests. Computer programmes have been developed for easy implements of these new confidence band based inferential methods. An illustrative example is provided.
\end{abstract}

Keywords: Confidence bands; Linear regression; Simultaneous inference; Statistical simulation. 


\section{Introduction}

Consider a standard multiple linear regression model given by

$$
\mathbf{Y}=X \boldsymbol{\beta}+\mathbf{e}
$$

where $\mathbf{Y}=\left(y_{1}, \cdots, y_{n}\right)^{T}$ is a vector of observations, $X$ is a $n \times(p+1)$ full column-rank design matrix with the first column given by $(1, \cdots, 1)^{T}$ and the $l$ th $(2 \leq l \leq p+1)$ column given by $\left(x_{1, l-1}, \cdots, x_{n, l-1}\right)^{T}, \boldsymbol{\beta}=\left(\beta_{0}, \cdots, \beta_{p}\right)^{T}$ is a vector of unknown coefficients, and $\mathbf{e}=\left(e_{1}, \cdots, e_{n}\right)^{T}$ is a vector of independent random errors with each $e_{i} \sim N\left(0, \sigma^{2}\right)$, where $\sigma^{2}$ is an unknown parameter.

One important problem for model (1.1) is to assess whether some of the coefficients $\beta_{i}$ 's are zero and so the corresponding covariates $x_{i}$ 's have no effect on the response variable $Y$. The model can therefore be simplified. To be specific, let $\boldsymbol{\beta}=\left(\boldsymbol{\beta}_{1}^{T}, \boldsymbol{\beta}_{2}^{T}\right)^{T}$, where $\boldsymbol{\beta}_{1}^{T}=$ $\left(\beta_{0}, \cdots, \beta_{p-k}\right)$ and $\boldsymbol{\beta}_{2}^{T}=\left(\beta_{p-k+1}, \cdots, \beta_{p}\right)$ with $1 \leq k \leq p$. If $\boldsymbol{\beta}_{2}$ is zero then the covariates $x_{p-k+1}, \cdots, x_{p}$ have no effect on the response variable $Y$ and model (1.1) reduces to

$$
\mathbf{Y}=X_{1} \boldsymbol{\beta}_{1}+\mathbf{e}
$$

where $X_{1}$ is formed by the first $p-k+1$ columns of the matrix $X$.

A commonly used statistical approach to assessing whether $\boldsymbol{\beta}_{2}$ is zero is to test the hypotheses

$$
H_{0}: \boldsymbol{\beta}_{2}=\mathbf{0} \text { against } H_{a}: \boldsymbol{\beta}_{2} \neq \mathbf{0}
$$

by using the partial $F$ test, which rejects $H_{0}$ if and only if

$$
\frac{[\text { Regression SS of model }(1.1)-\text { Regression SS of model }(1.2)] / k}{\text { MS residual of model (1.1) }}>f_{k, \nu}^{\alpha}
$$

where $f_{k, \nu}^{\alpha}$ is the upper $\alpha$ point of an $F$ distribution with $k$ and $\nu=n-(p+1)$ degrees of freedom. This can be found in most text books on multiple linear regression models; see e.g. Kleinbaum et al. (1998). 
The inferences that can be drawn from this partial $F$ test are that if $H_{0}$ is rejected then $\boldsymbol{\beta}_{2}$ is deemed to be non-zero and so at least some of the covariates $x_{p-k+1}, \cdots, x_{p}$ affect the response variable $Y$, and that if $H_{0}$ is not rejected then there is not enough statistical evidence to conclude that $\boldsymbol{\beta}_{2}$ is not equal to zero. (Unfortunately, this latter case is often mis-interpreted as $\boldsymbol{\beta}_{2}$ is equal to zero and so model (1.2) is accepted as more appropriate than model (1.1).) Note that, whether $H_{0}$ is rejected or not, no information on the magnitude of $\boldsymbol{\beta}_{2}$ is provided directly by this approach of hypotheses testing.

The first purpose of this paper is to show that there is a simultaneous confidence band associated naturally with the partial $F$ test and the partial $F$ test can be interpreted more intuitively via this simultaneous confidence band. The hypotheses (1.3) can in fact be tested by using this confidence band: the acceptance or rejection of $H_{0}$ is according to whether or not the zero hyper-plane lies completely inside the confidence band. The advantage of this confidence band approach over the partial $F$ test is that it provides information on the magnitude of $\beta_{p-k+1} x_{p-k+1}+\cdots+\beta_{p} x_{p}$, whether or not $H_{0}$ is rejected. This is discussed in Section 3. However, this confidence band is over the entire range $(-\infty, \infty)$ of each of the covariates $x_{p-k+1}, \cdots, x_{p}$. As a linear regression model is an acceptable approximation often only over a restricted region of these covariates, the part of the confidence band outside this restricted region is useless for inference. It is therefore unnecessary to guarantee the $1-\alpha$ simultaneous coverage probability over the entire range of each of these covariates. Furthermore, inferences deduced from the part of the confidence band outside the restricted region, such as the rejection of $H_{0}$, may not be valid since the assumed model may be wrong outside the restricted region after all. This calls for the construction of a $1-\alpha$ simultaneous confidence band only over this restricted region of the covariates. This confidence band is narrower and so allows more precise inferences than the confidence band associated with the partial $F$ test, and consequently induces an improved test of the hypotheses (1.3). These 
results are illuminated in Sections 4 and 5. Section 6 compares the powers of the partial $F$ test and the new test induced from the confidence band over a restricted region considered in Section 4. Some concluding remarks are contained in Section 7. But we first provide some preliminaries in Section 2.

\section{Some preliminaries}

The main result of this section is a simple algebraic result which we will use in Sections 3 and 4; it is related to the derivation of a simultaneous confidence band over the whole range of the covariates for one linear regression model (see, Miller, 1981, Chapter 2 Section 2). Denote the estimate of $\boldsymbol{\beta}$ by $\hat{\boldsymbol{\beta}}=\left(X^{T} X\right)^{-1} X^{T} \mathbf{Y} \sim \mathbf{N}\left(\boldsymbol{\beta}, \sigma^{2}\left(X^{T} X\right)^{-1}\right)$, and the estimate of $\sigma^{2}$ by $\hat{\sigma}^{2}=\|\mathbf{Y}-X \hat{\boldsymbol{\beta}}\|^{2} / \nu \sim \sigma^{2} \chi_{\nu}^{2} / \nu$ where $\nu=n-p-1$. It is well known that $\hat{\boldsymbol{\beta}}$ and $\hat{\sigma}^{2}$ are independent, and $\hat{\sigma}^{2}$ is the $M S$ residual of model (1.1). Let $\hat{\boldsymbol{\beta}}_{2}$ denote the estimate of $\boldsymbol{\beta}_{2}$. Clearly $\hat{\boldsymbol{\beta}}_{2}$ has the distribution $\mathbf{N}\left(\boldsymbol{\beta}_{2}, \sigma^{2} A\right)$, where $A$ is the $k \times k$ partition matrix from the last $k$ rows and the last $k$ columns of $\left(X^{T} X\right)^{-1}$. Since $A$ is symmetric and positive definite, there exists a unique symmetric positive definite matrix $W$ such that $A=W^{2}$. Denote the $i$ th column of $W$ by $\mathbf{w}_{i}$ for $i=1, \cdots, k$. Finally let $\mathbf{x}_{2}=\left(x_{p-k+1}, \cdots, x_{p}\right)^{T}$ denote the covariates corresponding to $\boldsymbol{\beta}_{2}$.

Lemma. For a hyper-rectangle region $C$ given by

$$
C=\left\{\mathbf{x}_{2}: a_{i}<x_{i}<b_{i}, i=p-k+1, \cdots, p\right\}
$$

where $-\infty \leq a_{i} \leq b_{i} \leq \infty$ for all $i=p-k+1, \cdots, p$ are given numbers, the following equation holds for any $\boldsymbol{\beta}_{2} \in R^{k}$

$$
\sup _{\mathbf{x}_{2} \in C} \frac{\left|\mathbf{x}_{2}^{T}\left(\hat{\boldsymbol{\beta}}_{2}-\boldsymbol{\beta}_{2}\right)\right|}{\sqrt{\operatorname{Var}\left(\mathbf{x}_{2}^{T} \hat{\boldsymbol{\beta}}_{2}\right)}}=\sup _{\mathbf{x}_{2} \in C} \frac{\left|\mathbf{x}_{2}^{T}\left(\hat{\boldsymbol{\beta}}_{2}-\boldsymbol{\beta}_{2}\right)\right|}{\sqrt{\sigma^{2} \mathbf{x}_{2}^{T} A \mathbf{x}_{2}}}=\|\mathbf{Z}\| \sup _{\mathbf{v} \in V} \frac{\left|\mathbf{v}^{T} \mathbf{Z}\right|}{\|\mathbf{v}\|\|\mathbf{Z}\|}
$$


where $\mathbf{Z}=W^{-1}\left(\hat{\boldsymbol{\beta}}_{2}-\boldsymbol{\beta}_{2}\right) / \sigma$ and $V=\left\{\mathbf{v}=W \mathbf{x}_{2}=x_{p-k+1} \mathbf{w}_{1}+\cdots+x_{p} \mathbf{w}_{k}: \mathbf{x}_{2} \in C\right\}$.

Proof. we have

$$
\sup _{\mathbf{x}_{2} \in C} \frac{\left|\mathbf{x}_{2}^{T}\left(\hat{\boldsymbol{\beta}}_{2}-\boldsymbol{\beta}_{2}\right)\right|}{\sqrt{\operatorname{Var}\left(\mathbf{x}_{2}^{T} \hat{\boldsymbol{\beta}}_{2}\right)}}=\sup _{\mathbf{x}_{2} \in C} \frac{\left|\left(W \mathbf{x}_{2}\right)^{T} W^{-1}\left(\hat{\boldsymbol{\beta}}_{2}-\boldsymbol{\beta}_{2}\right) / \sigma\right|}{\sqrt{\mathbf{x}_{2}^{T} A \mathbf{x}_{2}}}=\|\mathbf{Z}\| \sup _{\mathbf{x}_{2} \in C} \frac{\left|\left(W \mathbf{x}_{2}\right)^{T} \mathbf{Z}\right|}{\sqrt{\left(W \mathbf{x}_{2}\right)^{T}\left(W \mathbf{x}_{2}\right)}\|\mathbf{Z}\|}
$$

where this last term is just the right side of (2.1). It is noteworthy that $\mathbf{Z} \sim \mathbf{N}\left(\mathbf{0}, I_{k}\right)$ and that equation (2.1) holds for any $\boldsymbol{\beta}_{2} \in R^{k}$ and, in particular, for $\boldsymbol{\beta}_{2}=\mathbf{0}$.

The following are well known facts; see e.g. Scheffé (1959). The test statistic of the partial $F$ test has the alternative expression $\left\{\hat{\boldsymbol{\beta}}_{2}^{T} A^{-1} \hat{\boldsymbol{\beta}}_{2} / k\right\} / \hat{\sigma}^{2}$, i.e.

$$
\text { [Regression SS of model (1.1) - Regression SS of model (1.2)] }=\hat{\boldsymbol{\beta}}_{2}^{T} A^{-1} \hat{\boldsymbol{\beta}}_{2} \text {. }
$$

The random variate $\left\{\left(\hat{\boldsymbol{\beta}}_{2}-\boldsymbol{\beta}_{2}\right)^{T} A^{-1}\left(\hat{\boldsymbol{\beta}}_{2}-\boldsymbol{\beta}_{2}\right) / k\right\} / \hat{\sigma}^{2}$ has an $F$ distribution with degrees of freedom $k$ and $\nu$, and so

$$
P\left\{\frac{\left(\hat{\boldsymbol{\beta}}_{2}-\boldsymbol{\beta}_{2}\right)^{T} A^{-1}\left(\hat{\boldsymbol{\beta}}_{2}-\boldsymbol{\beta}_{2}\right) / k}{\text { MS residual of model }(1.1)} \leq f_{k, \nu}^{\alpha}\right\}=1-\alpha .
$$

\section{The Partial $F$ test from a confidence band viewpoint}

Note that $\boldsymbol{\beta}_{2}=\mathbf{0}$ if and only if $\mathbf{x}_{2}^{T} \boldsymbol{\beta}_{2}=0$ for all $\mathbf{x}_{2} \in R^{k}$. It is prudent that the usefulness of the covariates $\mathbf{x}_{2}$ in model (1.1) be judged by the magnitude of $\mathbf{x}_{2}^{T} \boldsymbol{\beta}_{2}$. To assess how close to zero $\mathbf{x}_{2}^{T} \boldsymbol{\beta}_{2}$ is, it is natural to use a simultaneous confidence band of the form

$$
\mathbf{x}_{2}^{T} \boldsymbol{\beta}_{2} \in \mathbf{x}_{2}^{T} \hat{\boldsymbol{\beta}}_{2} \pm c \hat{\sigma} \sqrt{\mathbf{x}_{2}^{T} A \mathbf{x}_{2}}, \forall x_{i} \in(-\infty, \infty) \text { for } i=p-k+1, \cdots, p
$$

where $c$ is the critical constant chosen so that the simultaneous coverage probability of this confidence band is equal to $1-\alpha$. This band is related to the Scheffé band for a whole linear regression model (see e.g. Miller, 1981, page 111). We now show that $c=\sqrt{k f_{k, \nu}^{\alpha}}$. 
The confidence level of the simultaneous band (3.1) is given by

$$
P\left\{\sup _{x_{i} \in(-\infty, \infty), i=p-k+1, \cdots, p} \frac{\left|\mathbf{x}_{2}^{T}\left(\hat{\boldsymbol{\beta}}_{2}-\boldsymbol{\beta}_{2}\right)\right|}{\hat{\sigma} \sqrt{\mathbf{x}_{2}^{T} A \mathbf{x}_{2}}} \leq c\right\} .
$$

Applying the lemma of Section 2 to the special case of $a_{i}=-\infty$ and $b_{i}=\infty$ for all $i=p-k+1, \cdots, p$, the supreme in the right side of (2.1) is equal to one since $\mathbf{v}$ can always be chosen to be in the same direction of $\mathbf{Z}$. This observation implies the probability (3.2) is equal to

$$
P\left\{\sqrt{\left(\hat{\boldsymbol{\beta}}_{2}-\boldsymbol{\beta}_{2}\right)^{T} A^{-1}\left(\hat{\boldsymbol{\beta}}_{2}-\boldsymbol{\beta}_{2}\right)} / \hat{\sigma} \leq c\right\}
$$

which, from $(2.3)$, is $1-\alpha$ if $c=\sqrt{k f_{k, \nu}^{\alpha}}$.

The relationship between the band (3.1) with $c=\sqrt{k f_{k, \nu}^{\alpha}}$ and the partial $F$ test can be seen from the following. The band (3.1) contains the zero hyper-plane $\mathbf{x}_{2}^{T} \mathbf{0}$ if and only if

$$
\sup _{x_{i} \in(-\infty, \infty), i=p-k+1, \cdots, p} \frac{\left|\mathbf{x}_{2}^{T} \hat{\boldsymbol{\beta}}_{2}\right|}{\hat{\sigma} \sqrt{\mathbf{x}_{2}^{T} A \mathbf{x}_{2}}} \leq c .
$$

This, by using the lemma of Section 2 for the special case of $a_{i}=-\infty, b_{i}=\infty$ for all $i=p-k+1, \cdots, p$ and $\boldsymbol{\beta}_{2}=\mathbf{0}$, is further equivalent to

$$
\frac{\sqrt{\hat{\boldsymbol{\beta}}_{2}^{T} A^{-1} \hat{\boldsymbol{\beta}}_{2}}}{\hat{\sigma}} \leq c \Longleftrightarrow \frac{\hat{\boldsymbol{\beta}}_{2}^{T} A^{-1} \hat{\boldsymbol{\beta}}_{2} / k}{\hat{\sigma}^{2}} \leq f_{k, \nu}^{\alpha}
$$

that is, the partial $F$ test does not reject the $H_{0}$ in (1.3) when using equation (2.2). In conclusion, If the band (3.1) contains the zero hyper-plane $\mathbf{x}_{2}^{T} \mathbf{0}$ then the partial $F$ test does not reject the $H_{0}$; if the band (3.1) excludes the zero hyper-plane $\mathbf{x}_{2}^{T} \mathbf{0}$ at one $\mathbf{x}_{2} \in R^{k}$ at least then the partial $F$ test rejects the $H_{0}$.

So the partial $F$ test can be regarded simply as a side product of the confidence band (3.1). More importantly the confidence band provides the information on the magnitude of $\mathbf{x}_{2}^{T} \boldsymbol{\beta}_{2}$ for every $\mathbf{x}_{2} \in R^{k}$, which is most suitable for judging whether $\mathbf{x}_{2}^{T} \boldsymbol{\beta}_{2}$ is small enough 
to be deleted from the original model (1.1) and hence to conclude that model (1.2) is more appropriate.

Note, however, in many real problems model (1.1) holds only in a certain range of the covariates. The null hypothesis $H_{0}$ in (1.3) should not be rejected simply because the zero hyper-plane goes out the confidence band for some $\mathbf{x}_{2}$ outside this range since the model is wrong outside this range. This brings up the problem of performing the test of (1.3) under constraints on the covariates, the focus of the next section.

\section{Confidence bands over a restricted region}

The confidence band (3.1) is over the entire range of each of the covariates $\mathbf{x}_{2}$, and so has some weaknesses. Firstly, a covariate may only take values in a restricted range. Secondly, even if a covariate may take values in the entire range $(-\infty, \infty)$, a linear regression model is seldom suitable for the entire range of the covariate. For example, a covariate such as age cannot take a negative value. Furthermore, it is inconceivable, for example, that a linear regression model that includes age as a covariate would hold for all values of age. So a simultaneous confidence band over a suitably chosen restricted region of the covariates $\mathbf{x}_{2}$ is more sensible. One should take into consideration the following points when choosing the restricted region. Firstly the regression model (1.1) should hold on the region. Secondly the region should be relevant to the interests of inference about the model. In standard clinical studies the values of the important covariates (such as age, blood pressure, etc.) are predefined by a set of inclusion/exclusion criteria; patients are randomized into the study only if they satisfy the inclusion criteria. Similarly, in survey studies, the range of the important covariates is fixed in advance as well, e.g., when considering risk factors for children the covariate age is usually constrained to lie within two thresholds. In these cases it may be 
natural to choose the pre-defined thresholds as the limits of the covariates.

The added incentive of a confidence band over a restricted region is that it is often narrower and hence allows more precise inferences over the restricted region than the confidence band over the entire range. In this section we consider the construction of the simultaneous confidence band

$$
\mathbf{x}_{2}^{T} \boldsymbol{\beta}_{2} \in \mathbf{x}_{2}^{T} \hat{\boldsymbol{\beta}}_{2} \pm c \hat{\sigma} \sqrt{\mathbf{x}_{2}^{T} A \mathbf{x}_{2}} \text { for all } \mathbf{x}_{2} \in C
$$

over a given rectangular region $C=\left\{\mathbf{x}_{2}: x_{i} \in\left(a_{i}, b_{i}\right),-\infty<a_{i} \leq b_{i}<\infty, i=p-k+\right.$ $1, \cdots, p\}$. It is clear as in Section 3 that the confidence band (4.1) induces a size $\alpha$ test of the hypotheses (1.3): $H_{0}$ is rejected if and only if the zero hyper-plane $\mathbf{x}_{2} \mathbf{0}$ is excluded from the band at one $\mathbf{x}_{2} \in C$ at least. The $p$-value of this test can be defined in the usual manner. This test is an improvement to the partial $F$ test in the sense that the partial $F$ test may reject $H_{0}$ due to the zero hyper-plane $\mathbf{x}_{2}^{T} \mathbf{0}$ is excluded from band (3.1) at some $\mathbf{x}_{2}$ outside $C$ where the assumed model (1.1) is false and hence the consequential inferences (e.g. confidence band) may be invalid. It is worth pointing out that this new test is also invariant under location and scale changes.

The central question is how the critical constant $c$ in (4.1) can be determined so that the simultaneous confidence level of this band is equal to $1-\alpha$. Note that, from the lemma of Section 2, the confidence level of the band (4.1) is given by $P\{T<c\}$ with

$$
T=\sup _{\mathbf{x}_{2} \in C} \frac{\left|\mathbf{x}_{2}^{T}\left(\hat{\boldsymbol{\beta}}_{2}-\boldsymbol{\beta}_{2}\right)\right|}{\hat{\sigma} \sqrt{\mathbf{x}_{2}^{T} A \mathbf{x}_{2}}}=\frac{\|\mathbf{Z}\|}{\hat{\sigma} / \sigma} \sup _{\mathbf{v} \in V} \frac{\left|\mathbf{v}^{T} \mathbf{Z}\right|}{\|\mathbf{v}\|\|\mathbf{Z}\|,}
$$

where $\mathbf{Z} \sim \mathbf{N}\left(\mathbf{0}, I_{k}\right), \hat{\sigma} / \sigma \sim \sqrt{\chi_{\nu}^{2} / \nu}$, and $\mathbf{Z}$ and $\hat{\sigma} / \sigma$ are independent random variables, and $V$ defined as in the lemma of Section 2. The distribution of $T$ does not depend on the unknown parameters $\boldsymbol{\beta}$ and $\sigma$, but it does depend on the region $C$ through the supreme over $V$ in (4.2) in a complicated way. 
Note that for the special case of $k=1$, which corresponds to testing whether the coefficient of one particular term of the model (1.1) is equal to zero, the supreme in (4.2) is clearly equal to one and so the critical constant $c$ is given by the usual $t$ value and no improvement over the usual $t$ test can be achieved by restricting the range of that covariate. Also, for the special case that the set $V$, which is a polyhedral cone, contains the origin $\mathbf{0}$ as an inner point, the supreme in (4.2) is again equal to one (by choosing a $\mathbf{v}$ to be in the same or opposite directions of $\mathbf{Z}$ ) and so the critical constant $c$ is given by $\sqrt{k f_{k, \nu}^{\alpha}}$ as the $F$ band (3.1).

In the general setting considered here, we propose to find the critical constant $c$ by using Monte Carlo simulation. This seems to be the only possible means, since it is clearly difficult to derive a useful formula for the distribution of $T$. We first simulate a pair of independent $\mathbf{Z}$ and $\hat{\sigma} / \sigma$. We then compute $T$ from (4.2). We repeat this to generate $R$ replicates of $T: T_{1}, \cdots, T_{R}$, and then use the $[(1-\alpha) R]$-th largest $T_{i}$ 's as an approximation of the critical constant $c$. An $R$ value of 100,000 usually produces an approximate $c$ value that is accurate to two decimal places at least; the readers are referred to Liu et al. (2005) for assessing the accuracy of a sample percentile as an approximation of the corresponding population percentile. The key in the simulation of each $T$ is to evaluate the supreme over $V$ in $(4.2)$ :

$$
Q=\sup _{\mathbf{v} \in V} \frac{\left|\mathbf{v}^{T} \mathbf{Z}\right|}{\|\mathbf{v}\|\|\mathbf{Z}\|}=\max \left\{Q_{1} \equiv \sup _{\mathbf{v} \in V} \frac{\mathbf{v}^{T} \mathbf{Z}}{\|\mathbf{v}\|\|\mathbf{Z}\|}, \quad Q_{2} \equiv \sup _{\mathbf{v} \in V} \frac{-\mathbf{v}^{T} \mathbf{Z}}{\|\mathbf{v}\|\|\mathbf{Z}\|}\right\} .
$$

Hereafter we consider methods to obtain $Q_{1}$; obviously, $Q_{2}$ is obtained similarly.

First, we can treat our problem as one of smooth optimization. Since any positive multiple of $\mathbf{v}$ leads to the same value of the objective function, it is not difficult to see that $Q_{1}$ can be obtained by solving the optimization problem

$$
\operatorname{maximize}_{\mathbf{v} \in \Re^{k}} \frac{\mathbf{v}^{T} \mathbf{Z}}{\|\mathbf{v}\|\|\mathbf{Z}\|}
$$


subject to $-W^{-1} \mathbf{v}+\gamma \mathbf{a} \leq 0, W^{-1} \mathbf{v}-\gamma \mathbf{b} \leq 0$, and $\gamma>0$

where $\mathbf{a}=\left(a_{p-k+1}, \cdots, a_{p}\right)^{T}$ and $\mathbf{b}=\left(b_{p-k+1}, \cdots, b_{p}\right)^{T}$. This is a smooth constrained optimization problem that can be solved, for example, by a gradient projection method with the inequality constraints handled by an active set strategy. One such algorithm for a similar problem, but in a different context, has been given and discussed in Liu, Jamshidian, and Zhang (2004).

Alternatively, realizing that the objective function in (4.3) is the cosine of the angle between $\mathbf{v}$ and $\mathbf{Z}$, it can be shown that the optimal value of $\mathbf{v}$ in (4.3) is equivalent to the optimal value of $\mathbf{v}$ that solves the following quadratic programming problem:

$$
\begin{aligned}
& \operatorname{minimize}_{\mathbf{v} \in \Re^{k}} \quad\|\mathbf{v}-\mathbf{Z}\|^{2} \\
& \text { subject to }-W^{-1} \mathbf{v}+\gamma \mathbf{a} \leq 0, W^{-1} \mathbf{v}-\gamma \mathbf{b} \leq 0, \text { and } \gamma>0
\end{aligned}
$$

provided that the solution to (4.4) is nonzero. If $\mathbf{Z} \in V$, then obviously the optimal value of (4.4) is $\mathbf{v}=\mathbf{Z}$ and it concurs with that of (4.3). In the case where $\mathbf{Z} \notin V$ and the optimal solution to (4.4) is zero, then it can be shown that $Q_{1}<0$ (i.e. cosine of the angle between $\mathbf{Z}$ and the closest vector in $V$ is negative). In such a case, the value of $Q$ is equal to $Q_{2}$ which is positive. Note that the value of $Q_{2}$ can be obtained via (4.4) with $\mathbf{Z}$ replaced by $-\mathbf{Z}$.

Approach (4.4) is preferable to (4.3) for two main reasons. First, the problem in (4.4) can be solved in a finite number of steps, whereas that in (4.3) requires iterative methods that do not have a finite termination property. Second, solving (4.4) always leads to the global minimum whereas solving (4.3) can lead to local maxima that may not be global. This latter point has been discussed in some detail in Liu et al. (2005). The quadratic programming problem (4.4) can be handled efficiently by an active set methodology similar 
to the algorithm given in Liu et al. (2005). Note however Liu et al.'s (2005) method deals with a quadratic programming problem whose objective function is positive definite. But the objective function of the quadratic program (4.4) has a positive semi-definite Hessian, since $\gamma$ does not appear in the objective function and hence the second derivative of the objective with respect to $\gamma$ is zero. The required modifications for positive semi-definiteness are discussed in Fletcher (1987, Chapter 10, Section 4). Alternatively, one may use readily available software that solves such quadratic programming problems (see e.g., http://wwwfp.mcs.anl.gov/otc/Guide/OptWeb/continuous/constrained/qprog/). We have developed a Matlab program for solving (4.4) and finding the critical constant $c$ in (4.1); this program is used for the computations in the next two sections.

\section{An Example}

In this section we use the aerobic fitness data from the SAS/STAT User's Guide (1990, p. 1443) to illustrate our methodology. In particular we will show that at significance level $\alpha=5 \%$ our test with bounds on the covariates rejects $H_{0}$ in $(1.3)$, whereas the partial $F$ test does not reject $H_{0}$.

The aerobic fitness data set consists of measurements on men involved in a physical fitness course at North Carolina State University. The variables measured are Age (years), Weight (kg), Oxygen intake rate (ml per $\mathrm{kg}$ body weight per minute), time to run 1.5 miles (minutes), heart rate while resting, heart rate while running (same time Oxygen rate measured), and maximum heart rate recorded while running. In a regression analysis of these data, the effect of age, weight, time to run 1.5 miles, heart rate while running and maximum heart rate on the oxygen intake rate was of interest. The SAS output for fitting 
the corresponding model

$$
\mathrm{OXY}=\beta_{0}+\beta_{1} \text { age }+\beta_{2} \text { weight }+\beta_{3} \text { runtime }+\beta_{4} \text { runpulse }+\beta_{5} \text { maxpulse }+\epsilon
$$

is given in Table 1. The coefficients of weight $\left(\beta_{2}\right)$ and maxpulse $\left(\beta_{5}\right)$ are the two least significant parameters. If no restriction is imposed on the covariates, then the critical value for the confidence band of $\beta_{2}$ weight $+\beta_{5}$ maxpulse with $95 \%$ confidence is $c=\sqrt{2 f_{2,25}^{0.05}}=$ 2.60 , with the upper and lower bands given by

$$
\begin{aligned}
& -.0723 \times \text { weight }+.3049 \times \text { maxpulse } \pm \\
& 2.60 \times \sqrt{.0533^{2} \times \text { weight }+.1339^{2} \times \text { maxpulse }-.002704 \times \text { weight } \times \text { maxpulse }} .
\end{aligned}
$$

These bands along with the zero plane are depicted in Figure 1a. From Figure 1a, these bands do not intersect the zero plane, and so the corresponding partial $\mathrm{F}$ test does not reject the null hypothesis $H_{0}: \beta_{2}=\beta_{5}=0$ against $H_{a}$ : not $H_{0}$. Obviously, negative values for weight and maxpulse have no physical meaning, and there is no reason to consider them in the analysis. In fact, the observed values for weight are in the interval $[59,91.6]$ and those for maxpulse are in the interval $[155,192]$. Figure 1b shows the portion of the Figure 1a restricted to the range of the observed values. Obviously the zero plane sits in between the lower and the upper band without intersecting them.

As we have argued in this paper, one may obtain sharper inferences if the covariates are restricted to their reasonable range. To this end, we calculated confidence bands for $\beta_{2}$ weight $+\beta_{5}$ maxpulse, using the simulation procedure outlined in Sections 4. More specifically, we restricted the covariates weight and maxpulse to their respective observed 
Table 1: Mori: please reformat the table in latex and retain only relevant infoSAS output for Model (5.1).

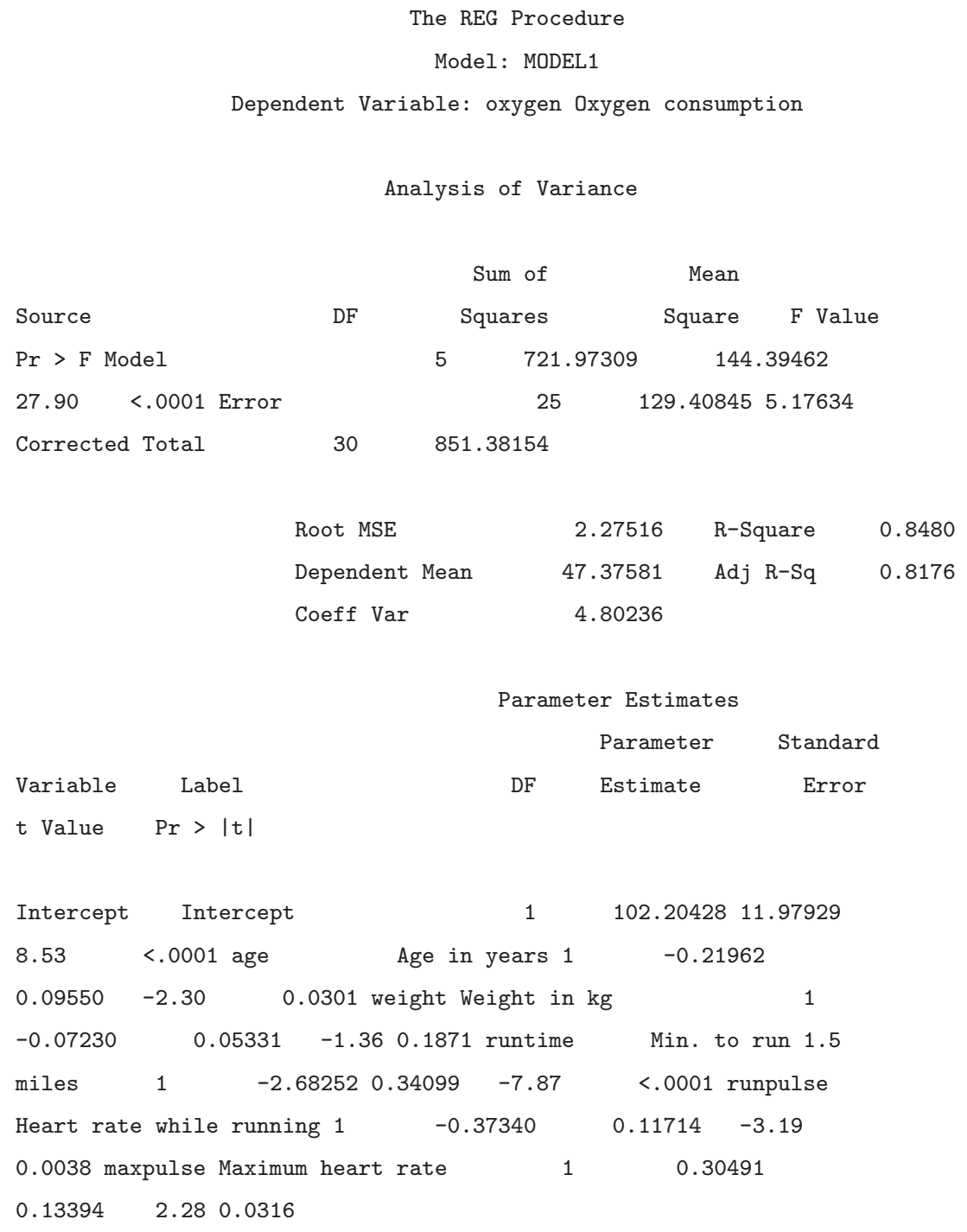


intervals mentioned above. The required critical value with 95\% confidence, using 100,000 simulations, is $c=2.11$. Thus the lower and upper confidence bands are the same as those in (5.2) except that the critical value 2.60 is replaced by 2.11 and that the covariates are restricted to their observed range. Figure 2 depicts this band along with the zero plane. Interestingly, the lower confidence band intersects the zero plane at values of weight ranging from about 59 to 72, and values of maxpulse ranging from about 157 to 190. So the restricted confidence band rejects the the null hypothesis $H_{0}: \beta_{2}=\beta_{5}=0$, whereas the partial $\mathrm{F}$ test and equivalently the unrestricted bands does not reject $H_{0}$, at $5 \%$ level.

It is noteworthy that one may obtain the $p$-values corresponding to the partial $F$ test and the test induced by the restricted band. In fact, when $k \geq 3$, plots of confidence bands may not be easily available even though we may plot 2-dimensional slices of a band by fixing the values of certain covariates. The $p$-values may be the best choice to determine the result of the hypotheses tests. For the example given, the $p$-value corresponding to the partial $F$ test is 0.066 and that for the restricted band is 0.047 ; this of course agrees with our observations from the confidence bands plotted .

The confidence bands provide information additional to the rejection or acceptance of $H_{0}$. For example, the confidence band of the partial $F$ test in Figure 1a provides a plausible range of the component $\beta_{2}$ weight $+\beta_{5}$ maxpulse: any $\beta_{2}$ weight $+\beta_{5}$ maxpulse that falls completely inside the band is a plausible component of the model. This information allows us to delete the component from the overall model (5.1) if the range restricts the component fairly close to the zero plane or to force us keeping the component in the overall model (5.1) if the range implies that the component can be marked away from the zero plane. The partial $F$ test is not suitable for this purpose of model selection, whether $H_{0}$ is rejected or not. The 
restricted confidence band in this example also tells us the range of the covariates for which the difference from zero of the component is more pronounced.

In this example, the region $C$ is taken as the observed region of the covariates weight and maxpulse. Note, however, if the covariates are already centered then this choice of $C$ will result in a $V$ that contains the origin as an inner point and so the critical constant $c$ is the same as the one in the $F$ band (3.1). On the other hand, this choice of $C$ is sensible only if we are not interested in comparisons between athletes.

\section{A power comparison}

It is clear from Section 3 that the partial $F$ test requires implicitly that model (1.1) holds over the entire range of the covariates $\mathbf{x}_{2}$, which is seldom true for any real problem. In this section we ignore this weakness of the partial $F$ test and compare the powers of the partial $F$ test and the new test induced by the band (4.1) for testing hypotheses (1.3).

It is well known that the power of the partial $F$ test is given by $P\left\{F_{\delta, k, \nu}>f_{k, \nu}^{\alpha}\right\}$ where $F_{\delta, k, \nu}$ denotes a non-central $F$ random variable with degrees of freedom $k$ and $\nu$ and non-central parameter $\delta=\sqrt{\boldsymbol{\beta}_{2}^{T} A^{-1} \boldsymbol{\beta}_{2} / \sigma^{2}}=\left\|W^{-1} \boldsymbol{\beta}_{2} / \sigma\right\|$. In particular, the power depends on $W, \boldsymbol{\beta}_{2}$ and $\sigma$ only through $\delta$. It is also known that the partial $F$ test is uniformly most powerful in the class of tests whose power depends on $\delta$ only. See e.g. Scheffé (1959).

The power of the new test is given by

$$
P\left\{\frac{\|\mathbf{Z}\|}{\hat{\sigma} / \sigma} \sup _{\mathbf{v} \in V} \frac{\left|\mathbf{v}^{T} \mathbf{Z}\right|}{\|\mathbf{v}\|\|\mathbf{Z}\|}>c\right\}
$$

where $V$ is defined in the lemma of Section $2, \mathbf{Z}=W^{-1} \hat{\boldsymbol{\beta}}_{2} / \sigma \sim \mathbf{N}\left(W^{-1} \boldsymbol{\beta}_{2} / \sigma, I_{k}\right), \hat{\sigma} / \sigma \sim$ $\sqrt{\chi_{\nu}^{2} / \nu}$, and $\mathbf{Z}$ and $\hat{\sigma} / \sigma$ are independent random variables. So the power depends on $C$, $W^{-1}$ and $\boldsymbol{\beta}_{2} / \sigma$ through $V$ and $W^{-1} \boldsymbol{\beta}_{2} / \sigma$. Note, in particular, the power depends on $V$ and 
$W^{-1} \boldsymbol{\beta}_{2} / \sigma$ only through the size of the polyhedral cone $V$ (which can be measured by the angles between the opposite faces of $V$ ) and the position of the mean vector $E(\mathbf{Z})=W^{-1} \boldsymbol{\beta}_{2} / \sigma$ relative to the cone $V$. Without loss of generality we have used $W=I$ and $\sigma=1$ in our simulation study.

We have performed an extensive simulation study to compare the power of the partial F test to that of the new test. For brevity we report a subset of our simulation studies that captures the gist of our findings. Specifically, we consider the cases $k=2,3$, and 4 with $\alpha=0.05$. For each of our simulation configurations that we describe below, the power of the partial $F$ test is readily calculated from the non-central $F$ distribution. The power of the new test is computed using (6.1). We estimate the critical constant $c$ in (6.1) by the simulation method of Section 4 using 100,000 replications. Once $c$ is obtained, we estimate the power from (6.1) by generating 15,000 copies of $\mathbf{Z}$ and $\hat{\sigma} / \sigma$, and determining the percentage of cases for which the inequality in (6.1) holds. Only 15,000 replications are used to approximate the power; this number should give a fairy accurate approximation since we are only estimating the probability of rejecting $H_{0}$.

For the case $k=2$ we have used $\nu=1,5,10$, and 100, and four $\delta$ values that correspond to a wide range of powers for the partial $\mathrm{F}$ test, namely $0.2,0.5,0.8$, and 0.95 . Figure 3 shows the three choices of $C$ (and the corresponding cones) that we have used in our simulations, denoted by $C_{1}, C_{2}$, and $C_{3}$. The corresponding cones $V$ of the $C$ 's have respectively angels of $5^{\circ}, 90^{\circ}$, and $170^{\circ}$ between the two edges. For example, $C_{2}$ comes from the restrictions $-\sqrt{2} / 2<x_{1}<\sqrt{2} / 2$ and $\sqrt{2} / 2<x_{2}<\sqrt{2}$, resulting in the $90^{\circ}$ cone shown. The essence of our simulation results for this case is shown in the first panel of Table 2 for $\nu=10$. 
For the case $k=3$, we have used $\nu=2,10$, and 100 and $\delta$ values that correspond to partial $\mathrm{F}$ test powers of $0.4, .85$, and .95 . Figure 4 shows one case where $C$ has the restrictions $-1 \leq x_{1} \leq 1,-.5 \leq x_{2} \leq .5$, and $0.5 \leq x_{3} \leq 1$. Note that the resulting cone $V$ can be controlled by the two angles between the two pairs of opposite faces of the cone. In our simulation study we have used various combinations of the two angles from small $5^{\circ}$, medium $90^{\circ}$, to large $170^{\circ}$. The result of the simulations for this case is illustrated in the second panel of Table 2 for $\nu=10$.

Finally, for the case $k=4$ we have used $\nu=1,5,10$, and 100 and two $\delta$ values corresponding to the partial F test powers of 0.4 and 0.9 . We have varied the cone $V$ based on three angles, mainly by generalizing the method that we used for $k=2$ and 3 . It is obviously not possible to draw the cone for this case. The results for this case are shown in the third panel of Table 2 for $\nu=10$.

As mentioned earlier, the power of the new test depends on the direction of $E(\mathbf{Z})=$ $W^{-1} \boldsymbol{\beta}_{2} / \sigma$ relative to the position of the cone $V$, and in particular for our case the direction of $\boldsymbol{\beta}_{2}$ (recall that we use $W=I$ and $\sigma=1$ ). To examine various positions of $\boldsymbol{\beta}_{2}$ relative to $V$ we have used the following directions for $\boldsymbol{\beta}_{2}^{T}$ :

\begin{tabular}{l|lll} 
& In & Mid & Orth \\
\hline$k=2$ & $(0,1)$ & $(1,1)$ & $(1,0)$ \\
$k=3$ & $(0,0,1)$ & $(1,1,1)$ & $(1,1,0)$ \\
$k=4$ & $(0,0,0,1)$ & $(1,1,1,1)$ & $(1,1,1,0)$
\end{tabular}

Note that the vectors in the directions of $(0,1),(0,0,1)$, and $(0,0,0,1)$ are in the "centers" of the cones $V$ that we have used for $k=2,3$ and 4, respectively (see e.g., Figures 3 and 4 for the cases $k=2,3)$. Thus, the three choices of $\boldsymbol{\beta}_{2}$ shown above represent $E(\mathbf{Z})$ in the "center" 
of $V$ (denoted by In), orthogonal to a vector in the center of $V$ (denoted by "Orth"), and in between these to extremes (denoted by "Mid").

It is clear from the table that, for a given $\delta$ value, while the power of the partial $F$ test is fixed, the power of the new test increases when the angle between the mean vector and the central direction of the cone decreases from $90^{\circ}$ to $0^{0}$. This phenomenon is more clear when the angles between the opposite faces of the cone $V$ become small. So when the angles between the opposite faces of the cone $V$ are small and the mean vector is close to the central direction of the cone, the new test usually has a large power that can be substantially greater than the power of the partial $F$ test. On the other hand, when the angles between the opposite faces of the cone $V$ are small and the mean vector is perpendicular to the central direction of the cone, the new test usually has a small power that can be considerably smaller than the power of the partial $F$ test. But when the angles between the opposite faces of the cone $V$ are large (close to $180^{\circ}$ ) the variation in the power of the new test, when the angle between the mean vector and the central direction of the cone changes, is small, and the powers of the new test are quite close to the power of the partial $F$ test.

It is our view that the region $C$ should be determined by factors other than the power property of the test. For example, in standard clinical studies the values of the important covariates (such as age, blood pressure, etc.) are pre-defined by a set of inclusion/exclusion criteria; patients are randomized into the study only if they satisfy the inclusion criteria. Similarly, in survey studies, the range of the important covariates is also fixed in advance. When considering risk factors for children, for instance, the covariate age is usually constrained to lie within two thresholds. Hence the natural choices of the intervals $\left(a_{l}, b_{l}\right)$ are frequently known and fixed in advance of an experiment. Even if such thresholds are not available prior to an experiment, natural boundaries may still be available and should be used for more precise inferences. For example, it is reasonable to assume $0<$ age $<100$, 
Table 2: Power Comparison for the $k=2$ case. The numerical values in the body of the table correspond to the power of the new test.

\begin{tabular}{|c|c|c|c|c|c|c|c|c|c|}
\hline \multirow[b]{4}{*}{ F Power } & \multicolumn{9}{|c|}{ Degree of freedom $=1$} \\
\hline & \multicolumn{3}{|c|}{$V$ Angle $=5$} & \multicolumn{3}{|c|}{$V$ Angle $=90$} & \multicolumn{3}{|c|}{$V$ Angle $=170$} \\
\hline & \multicolumn{3}{|c|}{ Mean Vector Position } & \multicolumn{3}{|c|}{ Mean Vector Position } & \multicolumn{3}{|c|}{ Mean Vector Position } \\
\hline & In & Mid & Orth & In & Mid & Orth & In & Mid & Orth \\
\hline 0.200 & 0.297 & 0.219 & 0.059 & 0.208 & 0.208 & 0.170 & 0.202 & 0.201 & 0.206 \\
\hline 0.500 & 0.698 & 0.548 & 0.083 & 0.530 & 0.530 & 0.400 & 0.500 & 0.500 & 0.500 \\
\hline 0.850 & 0.967 & 0.891 & 0.125 & 0.869 & 0.861 & 0.726 & 0.842 & 0.844 & 0.838 \\
\hline 0.950 & 0.996 & 0.969 & 0.146 & 0.960 & 0.957 & 0.859 & 0.948 & 0.948 & 0.948 \\
\hline
\end{tabular}

\begin{tabular}{|c|c|c|c|c|c|c|c|c|c|}
\hline \multirow[b]{4}{*}{ F Power } & \multicolumn{9}{|c|}{ Degrees of freedom $=5$} \\
\hline & \multicolumn{3}{|c|}{$V$ Angle $=5$} & \multicolumn{3}{|c|}{$V$ Angle $=90$} & \multicolumn{3}{|c|}{$V$ Angle $=170$} \\
\hline & \multicolumn{3}{|c|}{ Mean Vector Position } & \multicolumn{3}{|c|}{ Mean Vector Position } & \multicolumn{3}{|c|}{ Mean Vector Position } \\
\hline & In & Mid & Orth & In & Mid & Orth & In & Mid & Orth \\
\hline 0.200 & 0.299 & 0.177 & 0.055 & 0.224 & 0.207 & 0.161 & 0.200 & 0.203 & 0.204 \\
\hline 0.500 & 0.669 & 0.427 & 0.059 & 0.542 & 0.522 & 0.389 & 0.497 & 0.493 & 0.503 \\
\hline 0.850 & 0.950 & 0.755 & 0.069 & 0.880 & 0.864 & 0.724 & 0.847 & 0.847 & 0.848 \\
\hline 0.950 & 0.990 & 0.892 & 0.077 & 0.964 & 0.960 & 0.861 & 0.950 & 0.950 & 0.950 \\
\hline
\end{tabular}

\begin{tabular}{|c|c|c|c|c|c|c|c|c|c|}
\hline \multirow[b]{4}{*}{ F Power } & \multicolumn{9}{|c|}{ Degreesoffreedom $=10$} \\
\hline & \multicolumn{3}{|c|}{$V$ Angle $=5$} & \multicolumn{3}{|c|}{$V$ Angle $=90$} & \multicolumn{3}{|c|}{$V$ Angle $=170$} \\
\hline & \multicolumn{3}{|c|}{ Mean Vector Position } & \multicolumn{3}{|c|}{ Mean Vector Position } & \multicolumn{3}{|c|}{ Mean Vector Position } \\
\hline & In & Mid & Orth & In & Mid & Orth & In & Mid & Orth \\
\hline 0.200 & 0.272 & 0.164 & 0.051 & 0.229 & 0.202 & 0.160 & 0.195 & 0.198 & 0.198 \\
\hline 0.500 & 0.638 & 0.390 & 0.057 & 0.537 & 0.518 & 0.396 & 0.507 & 0.500 & 0.501 \\
\hline 0.850 & 0.930 & 0.702 & 0.064 & 0.875 & 0.863 & 0.730 & 0.848 & 0.846 & 0.852 \\
\hline 0.950 & 0.986 & 0.854 & 0.070 & 0.962 & 0.959 & 0.875 & 0.949 & 0.951 & 0.948 \\
\hline
\end{tabular}

\begin{tabular}{|c|c|c|c|c|c|c|c|c|c|}
\hline \multirow[b]{4}{*}{ F Power } & \multicolumn{9}{|c|}{ Degreeoffreedom $=100$} \\
\hline & \multicolumn{3}{|c|}{$V$ Angle $=5$} & \multicolumn{3}{|c|}{$V$ Angle $=90$} & \multicolumn{3}{|c|}{$V$ Angle $=170$} \\
\hline & \multicolumn{3}{|c|}{ Mean Vector Position } & \multicolumn{3}{|c|}{ Mean Vector Position } & \multicolumn{3}{|c|}{ Mean Vector Position } \\
\hline & In & Mid & Orth & In & Mid & Orth & In & Mid & Orth \\
\hline 0.200 & 0.273 & 0.159 & 0.051 & 0.231 & 0.202 & 0.168 & 0.200 & 0.203 & 0.202 \\
\hline 0.500 & 0.606 & 0.366 & 0.060 & 0.546 & 0.504 & 0.399 & 0.498 & 0.501 & 0.502 \\
\hline 0.850 & 0.915 & 0.677 & 0.065 & 0.870 & 0.857 & 0.752 & 0.853 & 0.850 & 0.852 \\
\hline 0.950 & 0.977 & 0.826 & 0.070 & 0.958 & 0.955 & 0.889 & 0.953 & 0.950 & 0.950 \\
\hline
\end{tabular}


Table 3: Power Comparison for the $k=3$ case. The numerical values in the body of the table correspond to the power of the new test.

\begin{tabular}{|c|c|c|c|c|c|c|c|c|c|c|c|c|}
\hline \multirow[b]{4}{*}{ F Power } & \multicolumn{12}{|c|}{ Degrees of freedom $=2$} \\
\hline & \multicolumn{3}{|c|}{$V$ Angle $=5,5$} & \multicolumn{3}{|c|}{$V$ Angle $=5,90$} & \multicolumn{3}{|c|}{$V$ Angle $=5,170$} & \multicolumn{3}{|c|}{$V$ Angle $=85,85$} \\
\hline & \multicolumn{3}{|c|}{ Mean Vector Position } & \multicolumn{3}{|c|}{ Mean Vector Position } & \multicolumn{3}{|c|}{ Mean Vector Position } & \multicolumn{3}{|c|}{ Mean Vector Position } \\
\hline & In & Mid & Orth & In & Mid & Orth & In & Mid & Orth & In & Mid & Orth \\
\hline 0.400 & 0.730 & 0.408 & 0.072 & 0.522 & 0.408 & 0.296 & 0.451 & 0.424 & 0.350 & 0.398 & 0.400 & 0.388 \\
\hline 0.850 & 0.992 & 0.861 & 0.106 & 0.941 & 0.861 & 0.683 & 0.891 & 0.879 & 0.772 & 0.852 & 0.855 & 0.838 \\
\hline 0.950 & 0.999 & 0.951 & 0.130 & 0.988 & 0.960 & 0.950 & 0.968 & 0.950 & 0.950 & 0.951 & 0.951 & 0.950 \\
\hline
\end{tabular}

\begin{tabular}{|c|c|c|c|c|c|c|c|c|c|c|c|c|}
\hline \multirow[b]{4}{*}{ F Power } & \multicolumn{12}{|c|}{ Degrees of freedom $=10$} \\
\hline & \multicolumn{3}{|c|}{$V$ Angle $=5,5$} & \multicolumn{3}{|c|}{$V$ Angle $=5,90$} & \multicolumn{3}{|c|}{$V$ Angle $=5,170$} & \multicolumn{3}{|c|}{$V$ Angle $=85,85$} \\
\hline & \multicolumn{3}{|c|}{ Mean Vector Position } & \multicolumn{3}{|c|}{ Mean Vector Positions } & \multicolumn{3}{|c|}{ Mean Vector Positions } & \multicolumn{3}{|c|}{ Mean Vector Position } \\
\hline & In & Mid & Orth & In & Mid & Orth & In & Mid & Orth & In & Mid & Orth \\
\hline 0.400 & 0.640 & 0.279 & 0.060 & 0.511 & 0.354 & 0.247 & 0.452 & 0.387 & 0.322 & 0.416 & 0.412 & 0.396 \\
\hline 0.850 & 0.970 & 0.660 & 0.073 & 0.920 & 0.794 & 0.602 & 0.883 & 0.850 & 0.746 & 0.861 & 0.855 & 0.842 \\
\hline 0.950 & 0.994 & 0.794 & 0.084 & 0.981 & 0.917 & 0.753 & 0.965 & 0.949 & 0.886 & 0.954 & 0.956 & 0.947 \\
\hline
\end{tabular}

\begin{tabular}{|c|c|c|c|c|c|c|c|c|c|c|c|c|}
\hline \multirow[b]{4}{*}{ F Power } & \multicolumn{12}{|c|}{ Degrees of freedom $=100$} \\
\hline & \multicolumn{3}{|c|}{$V$ Angle $=5,5$} & \multicolumn{3}{|c|}{$V$ Angle $=5,90$} & \multicolumn{3}{|c|}{$V$ Angle $=5,170$} & \multicolumn{3}{|c|}{$V$ Angle $=85,85$} \\
\hline & \multicolumn{3}{|c|}{ Mean Vector Position } & \multicolumn{3}{|c|}{ Mean Vector Position } & \multicolumn{3}{|c|}{ Mean Vector Position } & \multicolumn{3}{|c|}{ Mean Vector Position } \\
\hline & In & Mid & Orth & In & Mid & Orth & In & Mid & Orth & In & Mid & Orth \\
\hline 0.400 & 0.569 & 0.250 & 0.062 & 0.487 & 0.330 & 0.227 & 0.452 & 0.380 & 0.316 & 0.404 & 0.394 & 0.385 \\
\hline 0.850 & 0.944 & 0.575 & 0.073 & 0.910 & 0.753 & 0.564 & 0.880 & 0.837 & 0.745 & 0.854 & 0.855 & 0.850 \\
\hline 0.950 & 0.985 & 0.722 & 0.079 & 0.973 & 0.890 & 0.726 & 0.963 & 0.944 & 0.885 & 0.953 & 0.950 & 0.947 \\
\hline
\end{tabular}


Table 4: Power Comparison for the $k=4$ case. The numerical values in the body of the table correspond to the power of the new test.

\begin{tabular}{|c|c|c|c|c|c|c|}
\hline \multirow[b]{4}{*}{ F Power } & \multicolumn{6}{|c|}{ Degrees of freedom $=1$} \\
\hline & \multicolumn{3}{|c|}{$V$ Angle $=5,5,5$} & \multicolumn{3}{|c|}{$V$ Angle $=45,45,45$} \\
\hline & \multicolumn{3}{|c|}{ Mean Vector Position } & \multicolumn{3}{|c|}{ Mean Vector Position } \\
\hline & In & Mid & Orth & In & Mid & Orth \\
\hline 0.400 & 0.720 & 0.461 & 0.101 & 0.471 & 0.453 & 0.340 \\
\hline 0.900 & 0.999 & 0.943 & 0.244 & 0.953 & 0.940 & 0.816 \\
\hline
\end{tabular}

\begin{tabular}{|c|c|c|c|c|c|c|}
\hline \multirow[b]{4}{*}{ F Power } & \multicolumn{6}{|c|}{ Degrees of freedom $=5$} \\
\hline & \multicolumn{3}{|c|}{$V$ Angle $=5,5,5$} & \multicolumn{3}{|c|}{$V$ Angle $=45,45,45$} \\
\hline & \multicolumn{3}{|c|}{ Mean Vector Position } & \multicolumn{3}{|c|}{ Mean Vector Position } \\
\hline & In & Mid & Orth & In & Mid & Orth \\
\hline 0.400 & 0.760 & 0.313 & 0.065 & 0.537 & 0.410 & 0.288 \\
\hline 0.900 & 0.997 & 0.900 & 0.099 & 0.959 & 0.920 & 0.770 \\
\hline
\end{tabular}

\begin{tabular}{|c|c|c|c|c|c|c|}
\hline \multirow[b]{4}{*}{ F Power } & \multicolumn{6}{|c|}{ Degrees of freedom $=10$} \\
\hline & \multirow{2}{*}{\multicolumn{3}{|c|}{$V$ Angle $=5,5,5$}} & \multicolumn{3}{|c|}{$V$ Angle $=45,45,45$} \\
\hline & & & & & Vector 1 & \\
\hline & In & Mid & Orth & In & Mid & Orth \\
\hline 0.400 & 0.710 & 0.274 & 0.062 & 0.546 & 0.400 & 0.278 \\
\hline 0.900 & 0.992 & 0.679 & 0.091 & 0.959 & 0.908 & 0.763 \\
\hline
\end{tabular}

\begin{tabular}{|c|c|c|c|c|c|c|}
\hline \multirow[b]{4}{*}{ F Power } & \multicolumn{6}{|c|}{ Degrees of freedom $=100$} \\
\hline & \multicolumn{3}{|c|}{$V$ Angle $=5,5,5$} & \multicolumn{3}{|c|}{$V$ Angle $=45,45,45$} \\
\hline & \multicolumn{3}{|c|}{ Mean Vector Position } & \multicolumn{3}{|c|}{ Mean Vector Position } \\
\hline & In & Mid & Orth & In & Mid & Orth \\
\hline 0.400 & 0.631 & 0.222 & 0.062 & 0.528 & 0.367 & 0.250 \\
\hline 0.900 & 0.978 & 0.578 & 0.075 & 0.953 & 0.876 & 0.736 \\
\hline
\end{tabular}


$50<$ rest pulse $<100$, and so on.

For a chosen region $C$, it is clear from this power study for what alternative hypothesis $\boldsymbol{\beta}_{2} \neq \mathbf{0}$ the new test tends to be more (less) powerful than the partial $F$ test. Neither test is always more powerful than the other. But of course this observation/power study ignores the fact the partial $F$ test requires implicitly that model (1.1) holds over the entire range of the covariates $\mathbf{x}_{2}$ which is difficult to justify for any real problem, while the new test only requires that model (1.1) holds over the chosen region $C$ of the covariates $\mathbf{x}_{2}$.

\section{Conclusions}

It is pointed out in this paper that the usual partial $F$ test has in fact a naturally associated confidence band, which is much more informative than the test itself. But this confidence band is over the entire range of all the covariates. As regression models are true often only over a restricted range of the covariates, the part of this confidence band outside this range is useless and to guarantee an overall $1-\alpha$ confidence level is wasteful of resources. A narrower and hence more efficient confidence band is constructed over a restricted range of the covariates.

The side product of this confidence band is a new test of the hypotheses (1.3). This test is an improvement over the partial $F$ test in the sense that the partial $F$ test requires implicitly that model (1.1) holds over the entire range of the covariates $\mathbf{x}_{2}$ while the new test only requires that model $(1.1)$ holds over $\mathbf{x}_{2} \in C$. Ignoring this weakness of the partial $F$ test, the power comparison between these two tests indicates for what alternative hypothesis $\boldsymbol{\beta} \neq 0$ the new test can be either dramatically more or less powerful than the partial $F$ test. It is our view that the prime factors in choosing the region $C$ should be that model (1.1) holds on $C$ and that $C$ should be pertinent to the interests of inference about the model, 
rather than the power property of the new test. The confidence bands are more informative than the tests to allow us to make informed decision in model selection.

In this paper the covariates are assumed to be continuous variables and there is no functional relationship among them. If some covariates are discrete variables or there are functional relationships among the covariates the confidence band approach advocated here can be adapted in a natural way via the region $C$ in (4.1) and the corresponding $V$ in (4.2). The partial $F$ test approach simply throws away this kind of information completely.

Finally, the covariate region $C$ considered in this paper is restricted to a hyperrectangle, with the limits $a_{i}$ and $b_{i}$ being any real values. There may be situations that an ellipsoidal covariate region is of interest. But it is not clear how to construct a simultaneous

confidence band over a given ellipsoidal covariate region in general. Casella and Strawderman (1980) considered the construction of a simultaneous confidence band for a whole linear regression model over an ellipsoidal covariate region that only has a particular center and a particular shape. Further research in this direction is required.

Acknowledgements: We would like to thank two anonymous referees for many helpful comments.

\section{REFERENCES}

Casella, G. and Strawderman, W.E. (1980). Confidence bands for linear-regre -ssion with restricted predictor variables. J. Amer. Stat. Assoc. 75, 862-868.

Fletcher, R. (1987). Practical methods of Optimization, Wiley: New York.

Kleinbaum, D.G., Kupper, L.L., Muller, K.E. and Nizam, A. (1998). Applied Regression Analysis and Other Multivariable Methods, 3rd Edition. Duxbury Press. 
Liu, W., Jamshidian, M., and Zhang, Y. (2004). Multiple comparison of several linear regression models. J. of the American Statistical Association, 99, 395-403.

Liu, W., Jamshidian, M., Zhang, Y. and Donnelly, J. (2005). Simulation-based simultaneous confidence bands for a multiple linear regression model when the covariates are constrained. J. of Computational and Graphical Statistics, to appear.

Miller, R.G. (1981), Simultaneous Statistical Inference, Springer-Verlag.

SAS Institute Inc. (1990). SAS/STAT User's Guide, Version 6, Fourth edition, Volume 2 SAS InstituteInc., Cary, NC.

Scheffé, H. (1959). The Analysis of Variance. Wiley. 